\title{
Low extracellular lysyl oxidase expression is associated with poor prognosis in patients with prostate cancer
}

\author{
WEI ZHENG $^{1 *}$, XUEJIAN WANG $^{1 *}$, QIWEI CHEN ${ }^{1 *}$, KUN FANG $^{1}$, LINA WANG $^{1}$, FENG CHEN $^{1}$, \\ XIANCHENG LI ${ }^{1}$, ZIYAO LI ${ }^{1}$, JIANBO WANG ${ }^{1}$, YINGXI LIU ${ }^{2}$, DEYONG YANG ${ }^{1,2}$ and XISHUANG SONG ${ }^{1}$ \\ ${ }^{1}$ Department of Urology, First Affiliated Hospital of Dalian Medical University, Dalian, Liaoning 116011; \\ ${ }^{2}$ Department of Biomedical Engineering, Dalian University of Technology, Dalian, Liaoning 116024, P.R. China
}

Received June 12, 2015; Accepted July 22, 2016

DOI: $10.3892 / \mathrm{ol} .2016 .5118$

\begin{abstract}
Remodeling of the extracellular matrix (ECM), which is induced by lysyl oxidase (LOX), has been demonstrated to accompany tumor progression; however, the association between LOX expression levels and the malignant behavior of prostate cancer (Pca) remains unclear. The present study aimed to analyze the tumor-associated expression profile of LOX in patients with Pca and to evaluate its potential prognostic value. In the form of a retrospective study, the expression patterns of LOX and collagen I were analyzed in patients with benign prostate hyperplasia and Pca by immunohistochemical examination. The results demonstrated that, with the initiation and progression of Pca, the expression levels of LOX and collagen I were closely associated with Gleason score and tumor stage. In addition, although LOX was expressed in cancer and non-cancer tissues, the differential expression pattern observed in the ECM of Pca cells may indicate that LOX is an important molecule that affects the progression of this disease. Therefore, LOX expression level in the ECM may function as an independent predictor of Pca.
\end{abstract}

\section{Introduction}

Prostate cancer (Pca) is the most common malignancy affecting American men, with estimated 180,890 new cases and

Correspondence to: Professor Deyong Yang or Professor Xishuang Song, Department of Urology, First Affiliated Hospital of Dalian Medical University, 222 Zhongshan Road, Dalian, Liaoning 116011, P.R. China

E-mail: yangdeyong@dlmedu.edu.cn

E-mail: songxishuang@dlmedu.edu.cn

${ }^{*}$ Contributed equally

Abbreviations: $\mathrm{BPH}$, benign prostatic hyperplasia; Pca, prostate cancer; MMP, matrix metalloproteinase; ECM, extracellular matrix

Key words: prostate cancer, lysyl oxidase, extracellular matrix, prognosis
26,120 mortalities in 2015 (1). A number of studies have reported that remodeling of the tumor cell microenvironment-extracellular matrix (ECM) is closely associated with invasion and metastasis (2). However, the association between ECM remodeling and malignant behavior in Pca remains unclear.

The ECM is an omnipresent, three-dimensional structure located between tumor cells and their surrounding tissues (3). The function of the ECM is to provide more than just physical support for tissue elasticity and integrity, since the ECM is a flexible structure that is continuously remodeled to regulate tissue homeostasis (4). Collagen I is the most abundant ECM scaffolding protein, whose deposition, degradation and cross-linking affects cell growth and survival, and also promotes migration (5). In previous decades, a number of studies demonstrated that lysyl oxidase (LOX) and matrix metalloproteinases (MMPs) are crucial components of ECM remodeling (6-8). However, MMP inhibitors have failed clinically, indicating that other ECM remodeling parameters, including LOX, may instead regulate malignancy $(9,10)$.

LOX is a secreted, copper-dependent amine oxidase, which covalently cross-links elastin and collagen in the ECM (11-13). LOX is implicated in a number of pathological processes that affect the connective tissue $(14,15)$, including cancer progression and metastasis (16). LOX protein has been reported to possess intracellular and extracellular functions (17). Notably, previous studies have reported contradictory results regarding the expression of LOX in various forms of cancer (18). However, the associations between LOX expression, clinicopathological characteristics and Pca prognosis remain to be elucidated.

Therefore, the present study analyzed the expression of LOX in benign prostate hyperplasia (BPH) and Pca tissues, and compared the expression data with the pathological and clinical tumor features, and with patient prognosis. Furthermore, the current study aimed to explain the association between LOX and the malignant behavior of Pca.

\section{Patients and methods}

Patients and specimens. A total of 113 patients participated in the present study, with 36 patients having previously undergone transurethral resection of the prostate (TURP) or suprapubic transvesical prostatectomy for $\mathrm{BPH}$, and 77 patients having undergone TURP or radical prostatectomy for Pca. The mean 
Table I. Expression of LOX-cytoplasm, LOX-nuclear and LOX-ECM in BPH and Pca tissues.

\begin{tabular}{|c|c|c|c|c|c|c|c|c|c|c|c|c|c|c|c|c|}
\hline \multirow[b]{2}{*}{ Characteristic } & \multirow[b]{2}{*}{$\mathrm{N}$} & \multicolumn{4}{|c|}{ LOX-cytoplasm } & \multirow[b]{2}{*}{ P-value } & \multicolumn{4}{|c|}{ LOX-nuclear } & \multirow[b]{2}{*}{ P-value } & \multicolumn{4}{|c|}{ LOX-ECM } & \multirow[b]{2}{*}{ P-value } \\
\hline & & 0 & +1 & +2 & +3 & & 0 & +1 & +2 & +3 & & 0 & +1 & +2 & +3 & \\
\hline PH & 36 & 3 & 5 & 18 & 10 & 0.674 & 8 & 2 & 5 & 21 & 0.433 & 2 & 5 & 12 & 17 & 0.001 \\
\hline Pca & 77 & 5 & 11 & 32 & 24 & & 21 & 6 & 11 & 39 & & 15 & 31 & 19 & 12 & \\
\hline
\end{tabular}

Significant differences were observed between LOX-ECM in BPH and Pca tissues, as determined using the Mann-Whitney U test. LOX, lysyl oxidase; ECM, extracellular matrix; Pca, prostate cancer; BPH, benign prostate hyperplasia.

patient age was $72.3 \pm 7.8$ years, and the mean follow-up period was 33.1 months. None of the patients received chemotherapy or radiotherapy prior to surgery. The surgeries were performed at the Department of Urology, First Affiliated Hospital of Dalian Medical University (Dalian, China) between January 2007 and December 2009. The use of the samples was approved by the Ethics Committee of Dalian Medical University, and all patients provided written informed consent prior to surgery. Gleason scores were examined by two senior pathologists who were experienced in Pca diagnosis. The 36 patients with $\mathrm{BPH}$ formed the control group, while the Pca group was divided into two subgroups based on Gleason score (32 patients with Gleason score 2-7 and 45 patients with Gleason 8-10) and presence or absence of metastasis (50 patients, non-metastasis; and 27 patients, metastasis). The 2002 Tumor-Node-Metastasis classification system was used for pathological staging (19).

Immunohistochemical staining in human prostate tissues. Reagents, including anti-LOX (1:200; ab31238) and anti-collagen I (1:100; ab34710) polyclonal antibodies, were purchased from Abcam (Cambridge, UK). The BPH and Pca tissues (3-4 $\mu \mathrm{m}$ thick) were immediately fixed in $10 \%$ formalin, followed by paraffin embedding, deparaffinization and hydration. For antigen retrieval, all slides were placed in $10 \mathrm{mM}$ buffer (pH 9.0 LOX; pH 6.0 collagen I) and boiled for $20 \mathrm{~min}$. Subsequently, $3 \%$ hydrogen peroxide in methanol was used to destroy endogenous peroxidase activity. The tissue specimens were incubated with primary antibodies at room temperature for $1 \mathrm{~h}$, followed by incubation with the secondary horseradish peroxidase-conjugated anti-rabbit/mouse immunoglobulin G antibody (Santa Cruz Biotechnology, Inc., Dallas, TX, USA). For immunohistochemical staining, an EliVision ${ }^{\mathrm{TM}}$ Plus kit (Fuzhou Maixin Biotech Co., Ltd., Fuzhou, China) was used. The tissues were stained with 3,3'-diaminobenzidine and subsequently counterstained with hematoxylin.

Evaluation of immunostaining. The extent of expression of the target protein was calculated by two independent pathologists who were blinded to the clinicopathological features of the patients. For cytoplasm LOX assessment, staining intensity was scored as follows: 0 , negative; +1 , weak; +2 , medium; and +3 , strong. For nuclear LOX and for ECM LOX and collagen I assessment, the staining intensity was calculated by averaging five randomized microscopic fields, and was scored according to the percentage of positively stained cells out of the total number of tumor cells or matrix cells. The scoring system was as follows: $0,0 \% ;+1,1-25 \% ;+2,26-50 \%$; and $+3,>50 \%$.
Statistical analysis. SPSS software version 19.0 (IBM SPSS, Armonk, NY, USA) was used to perform the statistical analyses. Differences in LOX and collagen I expression in Pca and BPH tissues were assessed using the non-parametric Mann-Whitney $U$ test. The associations between LOX and collagen I expression in the ECM and the clinicopathological features of Pca were also assessed by the non-parametric Mann-Whitney U test. Survival functions and differences were calculated using the Kaplan-Meier method and assessed using the log-rank test. Associations between LOX-ECM expression and collagen I were assessed using Spearman's rank correlation coefficient and linear regression analysis, and multivariate survival analyses were performed using the Cox proportional hazards regression model. $\mathrm{P}<0.05$ was considered to indicate a statistically significant difference.

\section{Results}

Clinical and pathological variable analysis. Clinicopathological variables for the patients are summarized in Table I. A total of 77 patients with Pca who were between 53-86 years of age (mean, $72.3 \pm 7.8$ years) were included in the present study. According to the pathological classification for tumor staging, 33 patients with Pca were considered low grade and 44 patients were high grade. There were 32 low-to-medium grade cases (Gleason score, 2-7) and 45 high-grade cases (Gleason score, 8-10). Follow-up time ranged from 6 to 60 months (mean, 33.01 months).

LOX and collagen I expression in BPH and Pca. Immunohistochemistry was performed to detect the presence and location of LOX and collagen I expression in BPH and Pca tissues. The results demonstrated that LOX was expressed in the cytoplasm, nucleus and ECM (Table I), while collagen I was expressed in the ECM (Table II). The cytoplasmic and nuclear expression of LOX was not significantly different between BPH and Pca tissues $(\mathrm{P}=0.674$ and $\mathrm{P}=0.433$, respectively). A significant decrease was observed in LOX and collagen I expression in the ECM in the Pca tissues compared with the BPH tissues $(\mathrm{P}=0.001$; Table II).

LOX staining features in the ECM and Pca. The associations between clinical and pathological variables and LOX expression in the ECM are presented in Table III. No significant associations were observed between LOX expression and age $(\mathrm{P}=0.826)$, tumor metastasis $(\mathrm{P}=0.272)$ or prostate-specific 
Table II. Expression of collagen I in the ECM in BPH and Pca tissues.

\begin{tabular}{lcrrrrr}
\hline & & \multicolumn{4}{c}{ Collagen I } & \\
\cline { 3 - 5 } Characteristic & No. & 0 & +1 & +2 & +3 & P-value \\
\hline BPH & 36 & 1 & 2 & 6 & 27 & 0.001 \\
Pca & 77 & 6 & 45 & 23 & 3 & \\
\hline
\end{tabular}

Significant differences were observed between collagen I in BPH and Pca tissues, as determined by the Mann-Whitney U test. LOX, lysyl oxidase; ECM, extracellular matrix; $\mathrm{BPH}$, benign prostate hyperplasia; Pca, prostate cancer.

Table III. Association between clinicopathological parameters and LOX-ECM expression.

\begin{tabular}{|c|c|c|c|c|c|c|}
\hline \multirow[b]{2}{*}{ Variable } & \multirow[b]{2}{*}{ No. } & \multicolumn{4}{|c|}{ LOX-ECM } & \multirow[b]{2}{*}{ P-value } \\
\hline & & 0 & +1 & +2 & +3 & \\
\hline Age, years & & & & & & 0.826 \\
\hline$<72.3$ & 37 & 7 & 14 & 11 & 5 & \\
\hline$>72.3$ & 40 & 8 & 17 & 8 & 7 & \\
\hline Stage & & & & & & 0.004 \\
\hline T1-T2 & 33 & 3 & 12 & 10 & 8 & \\
\hline T3-T4 & 44 & 12 & 19 & 9 & 4 & \\
\hline Gleason score & & & & & & 0.004 \\
\hline $2-7$ & 32 & 4 & 10 & 9 & 9 & \\
\hline $8-10$ & 45 & 11 & 21 & 10 & 3 & \\
\hline Metastasis & & & & & & 0.272 \\
\hline No & 50 & 9 & 19 & 12 & 10 & \\
\hline Yes & 27 & 6 & 12 & 7 & 2 & \\
\hline PSA, ng/ml & & & & & & 0.540 \\
\hline$\leq 20$ & 29 & 5 & 11 & 8 & 5 & \\
\hline$>20$ & 48 & 10 & 20 & 11 & 7 & \\
\hline
\end{tabular}

LOX staining in the ECM was observed to be significantly associated with tumor stage $(\mathrm{P}=0.004)$ and Gleason score $(\mathrm{P}=0.004)$, as determined by the Mann-Whitney U test. LOX, lysyl oxidase; ECM, extracellular matrix; PSA, prostate-specific antigen.

antigen (PSA; $\mathrm{P}=0.54)$. However, LOX staining in the ECM was identified to be significantly associated with tumor stage $(\mathrm{P}=0.004)$ and Gleason score $(\mathrm{P}=0.004)$.

Association between LOX expression and cancer prognosis. Associations between overall survival time and LOX expression parameters were assessed by Kaplan-Meier survival analysis with a log-rank test. As presented in Fig. 1, the mean overall survival time significantly decreased in the low ECM LOX expression group compared with the high ECM LOX expression group (low LOX in ECM: Mean overall survival, 27.89 months; high LOX in ECM: Mean overall survival, 40.61 months; $\mathrm{P}=0.023$ ).

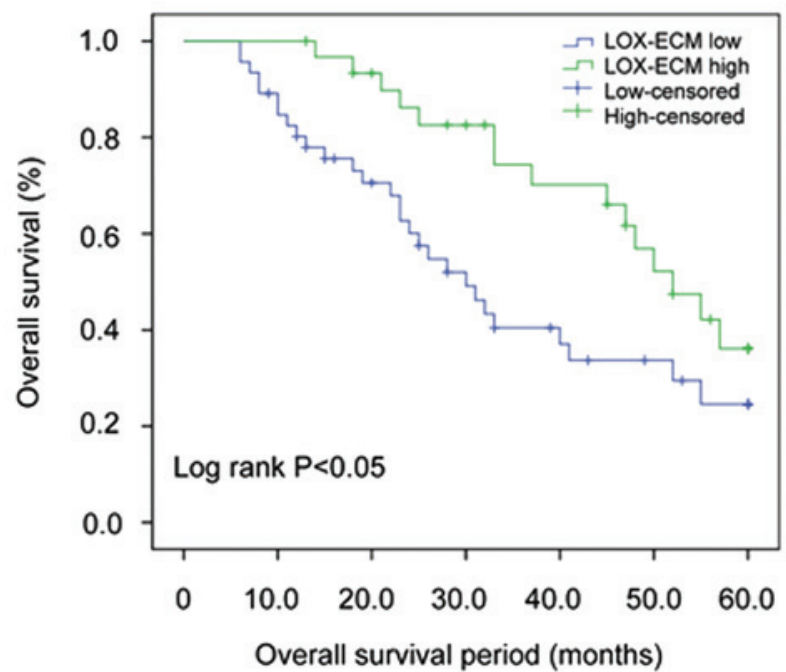

Figure 1. Kaplan-Meier estimates of cumulative overall survival time from LOX-ECM expression levels. Significant differences were observed between the high- and low-expression LOX-ECM groups, indicating the predictive value of LOX-ECM expression for prostate cancer. Log-rank test P-values are presented in the figure. LOX, lysyl oxidase; ECM, extracellular matrix.

A multivariate analysis was performed based on the Cox proportional hazards regression model that assessed the independent predictive value of LOX expression in the ECM, in addition to relevant clinical and pathological parameters, including tumor stage, age, Gleason score, PSA and distant metastasis. As presented in Table IV, decreased LOX expression in the ECM was identified as an independent prognostic marker for overall survival $(\mathrm{P}=0.032)$, in addition to tumor stage $(\mathrm{P}=0.005)$ and Gleason score $(\mathrm{P}=0.008)$.

Taken together, these results suggest that the expression of LOX in the ECM has a prognostic effect independent of Gleason score and tumor stage, and a decreased expression of LOX in the ECM predicts an increased risk of tumor-associated mortality.

Association between ECM LOX and collagen I expression. To investigate whether LOX expression in the ECM was associated with collagen I, the most important component of the ECM (20), the current study analyzed the expression of collagen I. As presented in Fig. 2, collagen I was primarily expressed in the ECM, and its expression levels were significantly reduced in the Pca tissues compared with the BPH tissues $(\mathrm{P}=0.001)$. The expression of collagen $\mathrm{I}$ in the ECM is in agreement with that of LOX, which suggests that they are closely associated. Spearman's rank correlation coefficient analysis identified a significant correlation between ECM LOX percentage and collagen I percentage $(r=0.722 ; \mathrm{P}=0.001)$.

\section{Discussion}

Over the last few years, a number of researchers have focused on the association between solid tumor ECM remodeling and malignant behavior $(21,22)$. Previously published results have indicated that the remodeling of the ECM is important in the progression of Pca (23-25). LOX is well known for its role in ECM remodeling, whereby the protein initiates cross-linking 
Table IV. Cox regression analysis of prognostic parameters for overall survival in patients with prostate cancer.

\begin{tabular}{lccccccc}
\hline Overall survival parameters & $\mathrm{B}$ & $\mathrm{SE}$ & Wald & $\mathrm{df}$ & P-value & HR & $95 \%$ CI \\
\hline LOX-ECM & -0.760 & 0.355 & 4.594 & 1 & $0.032^{\mathrm{a}}$ & 0.468 & $0.233-0.937$ \\
Gleason score & 1.175 & 0.440 & 7.117 & 1 & $0.008^{\mathrm{a}}$ & 3.238 & $1.366-7.675$ \\
Stage & 1.389 & 0.491 & 8.002 & 1 & $0.005^{\mathrm{a}}$ & 4.013 & $1.532-10.508$ \\
\hline
\end{tabular}

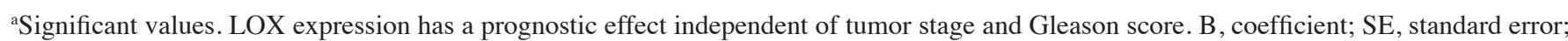
df, degrees of freedom; HR, hazard ratio; CI, confidence interval; LOX, lysyl oxidase; ECM, extracellular matrix.

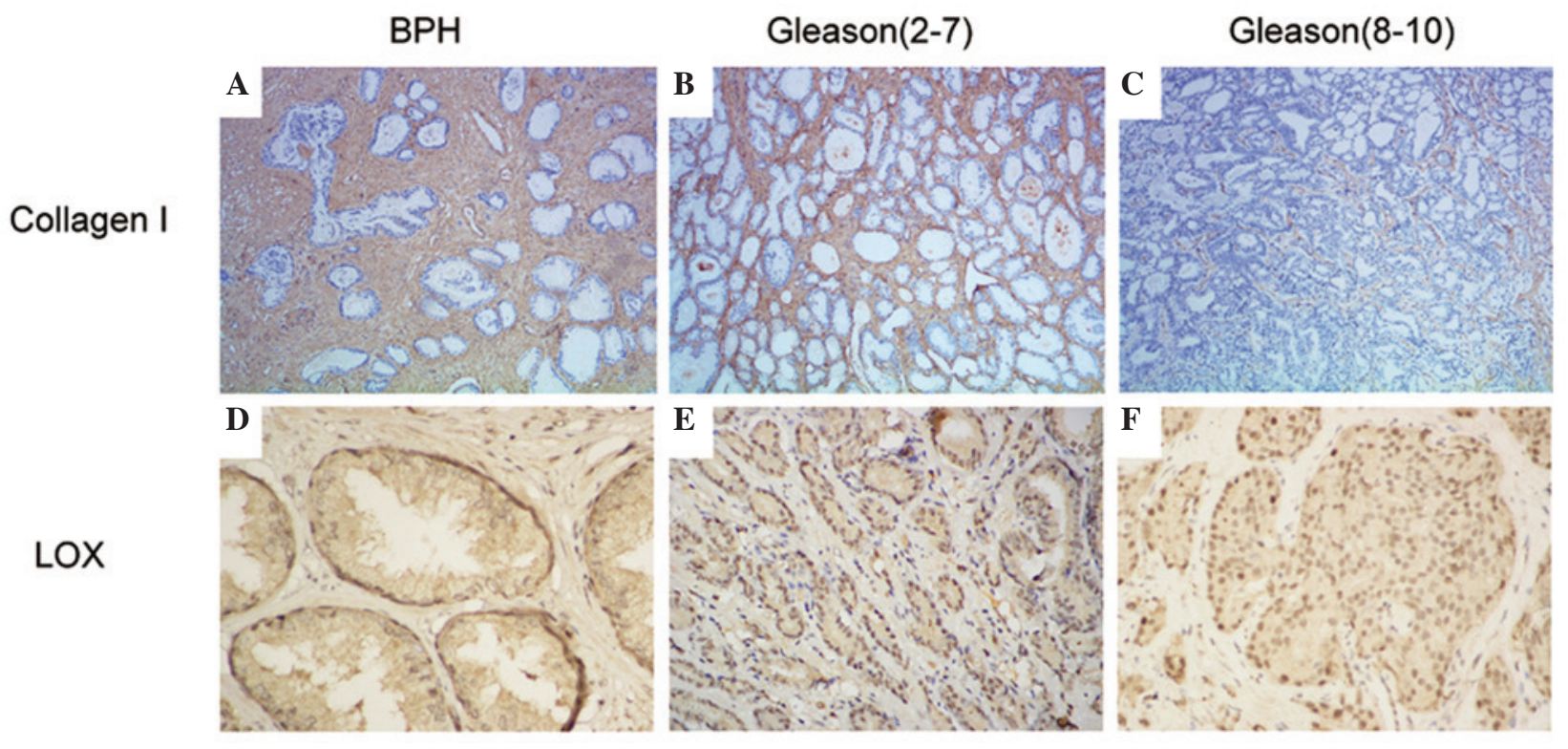

Figure 2. Immunohistochemical analysis of LOX and collagen I expression in BPH and Pca specimens. (A) Collagen I was primarily expressed in the ECM of BPH tissues. (B) Collagen I was expressed at low levels in the ECM of low-grade Pca tissues. (C) Collagen I expression was significantly reduced in the ECM of high-grade Pca tissues. (D) LOX was primarily expressed in the ECM of BPH tissues. (E) LOX was expressed at low levels in the ECM of low-grade Pca tissues. (F) LOX expression was significantly reduced in the ECM of high-grade Pca tissues. LOX, lysyl oxidase; BPH, benign prostate hyperplasia; Pca, prostate cancer; ECM, extracellular matrix. Magnification, x400.

of elastin and collagen I (26). However, LOX contradictory functions have been observed intra- and extracellularly in different solid malignancies; for instance, LOX enhanced breast cancer metastasis but had a inhibitory effect on hepatic and lung cancer (27). Thus, the current study analyzed the expression levels of LOX and collagen I in Pca and BPH tissues by immunohistochemistry, and aimed to determine the association between LOX and the malignant behavior of Pca.

Collagen I is the most abundant scaffold protein observed in the ECM, and through its organization, orientation and chemical modification, the malignant behavior of tumor cells may be altered (28). The mechanism of collagen remodeling has been studied in a number of solid tumors. For example, following the deposition and cross-linking of collagen induced by LOX, it was observed that phosphoinositide 3-kinase signaling was activated, which promoted invasion and tumor progression in breast cancer (29). However, in accordance with a previous study (30), the current study demonstrated that the expression of collagen I was significantly decreased in Pca tissues, particularly in advanced Pca, compared with
BPH tissues (Fig. 2A-C). These results demonstrate that the mechanism of ECM remodeling may vary in different malignancies.

It is widely understood that collagen cross-linking is primarily mediated by LOX and LOX-like enzymes, which are commonly upregulated in various forms of cancer and at metastatic sites, and that patients with high LOX expression have poor survival in comparison with those with low LOX expression (31). In the current study, it was observed that LOX exhibited a differential expression pattern between BPH and Pca tissues. In the BPH tissues, LOX was located in the cytoplasm, nucleus and ECM, whereas in the Pca tissues, a lower level of LOX was secreted into the ECM. In addition, the expression of LOX was higher in the low-grade Pca tissues compared with the high-grade Pca tissues (Fig. 2D-F). The expression patterns of LOX and collagen I corresponded to tumor stage and Gleason score (Table IV). These results demonstrate that the remodeling effect induced by LOX was present in the ECM in Pca tissues, which is in accordance with previous studies $(32,33)$. 
Multiple studies have reported that the LOX family functions intra- and extracellularly in cancer tissues (34). To maintain homeostasis and tissue stability, LOX is expressed intra- and extracellularly in normal tissues (35). When tissue suffers from injury or damage, the oxidative effect induced by LOX results in the cross-linking of collagen and elastin in what is known as a host response (36). This response is similar to the formation of a 'shell', repairing the damage and inhibiting tumor cell migration (37). Notably, a high expression of LOX was observed in basal cells, which functions as another form of 'shell' to maintain glandular structure integrity. In the present study, it was observed that, with the progression of Pca, LOX expression in the ECM significantly decreased. Subsequently, it was speculated that during the initial stages of Pca development, LOX may drive collagen to form a host response to repair the damage arising from tumorigenesis. However, with the progression of Pca, certain hydrolytic enzymes such as MMPs may inhibit this process through the degradation of collagen, resulting in a loss of the stimulatory element. This may be the reason that the expression of LOX decreases in the ECM with Pca progression.

To the best of our knowledge, LOX, MMPs and fibronectin are key molecules in the ECM remodeling signaling pathway $(38,39)$. Following the initiation of Pca, the tumor cells transform to acquire a malignant phenotype. Certain important proteases, including LOX and MMPs, are secreted from the intracellular space to the ECM, which may result in collagen deposition, cross-linking and degradation. These changes in components result in the remodeling of the cellular biomechanical microenvironment. The signal transduction of fibronectin inside tumor cells functions via integrins, which are molecular switches located on the cell membrane. In addition, the secretion of focal adhesion kinase and LOX strengthens the malignant phenotype. Simultaneously, due to limited space, hyperproliferative cancer cells compress against each other and generate tension that may result in cytoskeletal deformation. The change in the biomechanical properties of tumor cells may also affect the expression of integrins. Finally, with the simulation of ECM remodeling and biomechanical changes, the invasive and metastatic ability of Pca cells are likely to be enhanced (34,40-42).

In conclusion, the present study identified that LOX expression in the ECM is correlated with Pca stage, Gleason score and tumor-associated mortality. Expression of LOX protein also appeared to function as a marker for Pca prognosis. Thus, LOX may operate as a cancer suppressor in Pca. However, in vivo experiments are required to confirm the function of LOX in Pca detection. Furthermore, additional studies are warranted to further understand the mechanism of LOX in the regulation of Pca cell apoptosis, proliferation, invasion and metastasis, in addition to elucidate the association between LOX and the ECM.

\section{Acknowledgements}

The present study was supported by grants from the Youth Fund of the National Natural Science Foundation of China (Beijing, China; grant no. 81202030) and the National Natural Science Foundation of China (grant nos. 81271603 and 81372761$)$.

\section{References}

1. Winchester DA, Gurel B, Till C, Goodman PJ, Tangen CM, Santella RM, Johnson-Pais TL, Leach RJ, Thompson IM, $\mathrm{Xu} \mathrm{J}$, et al: Key genes involved in the immune response are generally not associated with intraprostatic inflammation in men without a prostate cancer diagnosis: Results from the prostate cancer prevention trial. Prostate 76: 565-574, 2016.

2. Bonnans C, Chou J and Werb Z: Remodelling the extracellular matrix in development and disease. Nat Rev Mol Cell Biol 15: 786-801, 2014.

3. Chester D and Brown AC: The role of biophysical properties of provisional matrix proteins in wound repair. Matrix Biol S0945-053X: 30185-30188, 2016.

4. Hynes RO: The extracellular matrix: Not just pretty fibrils. Science 326: 1216-1219, 2009.

5. Lo CM, Wang HB, Dembo M and Wang YL: Cell movement is guided by the rigidity of the substrate. Biophys J 79: 144-152, 2000.

6. Yamada S, Wang KY, Tanimoto A and Sasaguri Y: Novel function of histamine signaling in hyperlipidemia-induced atherosclerosis: Histamine $\mathrm{H} 1$ receptors protect and $\mathrm{H} 2$ receptors accelerate atherosclerosis. Pathol Int 65: 67-80, 2015.

7. Boufraqech M, Zhang L, Nilubol N, Sadowski SM, Kotian S, Quezado M and Kebebew E: Lysyl oxidase (LOX) transcriptionally regulates SNAI2 expression and TIMP4 secretion in human cancers. Clin Cancer Res 30, 2016 (Epub ahead of print]).

8. Palladini G, Ferrigno A, Richelmi P, Perlini S and Vairetti M: Role of matrix metalloproteinases in cholestasis and hepatic ischemia/reperfusion injury: A review. World J Gastroenterol 21: 12114-12124, 2015.

9. Coussens LM, Fingleton B and Matrisian LM: Matrix metalloproteinase inhibitors and cancer: Trials and tribulations. Science 295: 2387-2392, 2002.

10. Levental KR, Yu H, Kass L, Lakins JN, Egeblad M, Erler JT, Fong SF, Csiszar K, Giaccia A, Weninger W, et al: Matrix crosslinking forces tumor progression by enhancing integrin signaling. Cell 139: 891-906, 2009.

11. Kagan HM and Li W: Lysyl oxidase: Properties, specificity, and biological roles inside and outside of the cell. J Cell Biochem 88: 660-672, 2003

12. Vadasz Z, Kessler O, Akiri G, Gengrinovitch S, Kagan HM, Baruch Y, Izhak OB and Neufeld G: Abnormal deposition of collagen around hepatocytes in Wilson's disease is associated with hepatocyte specific expression of lysyl oxidase and lysyl oxidase like protein-2. J Hepatol 43: 499-507, 2005.

13. Hase H, Jingushi K, Ueda Y, Kitae K, Egawa H, Ohshio I, Kawakami R, Kashiwagi Y, Tsukada Y, Kobayashi T, et al: LOXL2 status correlates with tumor stage and regulates integrin levels to promote tumor progression in ccRCC. Mol Cancer Res 12: 1807-1817, 2014.

14. Cenizo V, André V, Reymermier C, Sommer P, Damour O and Perrier E: LOXL as a target to increase the elastin content in adult skin: A dill extract induces the LOXL gene expression. Exp Dermatol 15: 574-581, 2006.

15. Liu X, Zhao Y, Gao J, Pawlyk B, Starcher B, Spencer JA, Yanagisawa H, Zuo J and Li T: Elastic fiber homeostasis requires lysyl oxidase-like 1 protein. Nat Genet 36: 178-182, 2004.

16. Xiao Q and Ge G: Lysyl oxidase, extracellular matrix remodeling and cancer metastasis. Cancer Microenviron 5: 261-273, 2012.

17. Barker HE, Cox TR and Erler JT: The rationale for targeting the LOX family in cancer. Nat Rev Cancer 12: 540-552, 2012.

18. Erler JT, Bennewith KL, Nicolau M, Dornhöfer N, Kong C, Le QT, Chi JT, Jeffrey SS and Giaccia AJ: Lysyl oxidase is essential for hypoxia-induced metastasis. Nature 440: 1222-1226, 2006.

19. Chang SS and Amin MB: Utilizing the tumor-node-metastasis staging for prostate cancer: The sixth edition, 2002. CA Cancer J Clin 58: 54-59, 2008.

20. Liu W, Burdick JA and van Osch GJ: Plant-derived recombinant human collagen: A strategic approach for generating safe human ECM-based scaffold. Tissue Eng Part A 19: 1489-1490, 2013.

21. Alarab M, Kufaishi H, Lye S, Drutz H and Shynlova O: Expression of extracellular matrix-remodeling proteins is altered in vaginal tissue of premenopausal women with severe pelvic organ prolapse. Reprod Sci 21: 704-715, 2014.

22. Wood SL, Pernemalm M, Crosbie PA and Whetton AD: The role of the tumor-microenvironment in lung cancer-metastasis and its relationship to potential therapeutic targets. Cancer Treat Rev 40: 558-566, 2014. 
23. Taddei ML, Giannoni E, Comito G and Chiarugi P: Microenvironment and tumor cell plasticity: An easy way out. Cancer Lett 341: 80-96, 2013

24. Menen R, Pinney E, Hassanein MK, Kolostova K, Bobek V, Suetsugu A, Zhang N, Bouvet M, Naughton GK and Hoffman RM: Inhibition of metastasis of circulating human prostate cancer cells in the chick embryo by an extracellular matrix produced by foreskin fibroblasts in culture. Anticancer Res 32: 1573-1577, 2012.

25. Palumbo A Jr, Ferreira LB, Reis de Souza PA, Oliveira FL, Pontes B, Viana NB, Machado DE, Palmero CY, Alves LM, Gimba ER and Nasciutti LE: Extracellular matrix secreted by reactive stroma is a main inducer of pro-tumorigenic features on LNCaP prostate cancer cells. Cancer Lett 321: 55-64, 2012.

26. Przybyt E, van Luyn MJ and Harmsen MC: Extracellular matrix components of adipose derived stromal cells promote alignment, organization, and maturation of cardiomyocytes in vitro. J Biomed Mater Res A 103: 1840-1848, 2014.

27. Grimsby JL, Lucero HA, Trackman PC, Ravid K and Kagan HM: Role of lysyl oxidase propeptide in secretion and enzyme activity. J Cell Biochem 111: 1231-1243, 2010.

28. Barcus CE, Holt EC, Keely PJ,Eliceiri KW and Schuler LA: Dense collagen-I matrices enhance pro-tumorigenic estrogen-prolactin crosstalk in MCF-7 and T47D breast cancer cells. PLoS One 10: $\mathrm{e} 0116891,2015$.

29. Li Y, Zhao H, Wang Y, Zheng H, Yu W, Chai H, Zhang J, Falck JR, Guo AM, Yue J, et al: Isoliquiritigenin induces growth inhibition and apoptosis through downregulating arachidonic acid metabolic network and the deactivation of PI3K/Akt in human breast cancer. Toxicol Appl Pharmacol 272: 37-48, 2013.

30. Wang X, Wang J, Liu Y, Zong H, Che X, Zheng W, Chen F, Zhu Z, Yang D and Song X: Alterations in mechanical properties are associated with prostate cancer progression. Med Oncol 31: 876, 2014.

31. Erler JT, Bennewith KL, Cox TR, Lang G, Bird D, Koong A, Le QT and Giaccia AJ: Hypoxia-induced lysyl oxidase is a critical mediator of bone marrow cell recruitment to form the premetastatic niche. Cancer Cell 15: 35-44, 2009.

32. Liu J, Ping W, Zu Y and Sun W: Correlations of lysyl oxidase with MMP2/MMP9 expression and its prognostic value in non-small cell lung cancer. Int J Clin Exp Pathol 7: 6040-6047, 2014.
33. Takahashi T, Friedmacher F, Takahashi H, Daniel Hofmann A and Puri P: Lysyl oxidase expression is decreased in the developing diaphragm and lungs of nitrofen-induced congenital diaphragmatic hernia. Eur J Pediatr Surg 25: 15-19, 2015.

34. Cox TR, Bird D, Baker AM, Barker HE, Ho MW, Lang G and Erler JT: LOX-mediated collagen crosslinking is responsible for fibrosis-enhanced metastasis. Cancer Res 73: 1721-1732, 2013.

35. Wei L, Song XR, Sun JJ, Wang XW, Xie L and Lv LY: Lysyl oxidase may play a critical role in hypoxia-induced NSCLC cells invasion and migration. Cancer Biother Radiopharm 27: 672-677, 2012.

36. Tuinstra HM, Margul DJ, Goodman AG, Boehler RM, Holland SJ, Zelivyanskaya ML, Cummings BJ, Anderson AJ and Shea LD: Long-term characterization of axon regeneration and matrix changes using multiple channel bridges for spinal cord regeneration. Tissue Eng Part A 20: 1027-1037, 2014.

37. Burns-Cox N, Avery NC, Gingell JC and Bailey AJ: Changes in collagen metabolism in prostate cancer: A host response that may alter progression. J Urol 166: 1698-1701, 2001.

38. Kothapalli D, Liu SL, Bae YH, Monslow J, Xu T, Hawthorne EA, Byfield FJ, Castagnino P, Rao S, Rader DJ, et al: Cardiovascular protection by ApoE and ApoE-HDL linked to suppression of ECM gene expression and arterial stiffening. Cell Reports 2: $1259-1271,2012$

39. Helleman J, Jansen MP, Ruigrok-Ritstier K, van Staveren IL, Look MP, Meijer-van Gelder ME, Sieuwerts AM, Klijn JG, Sleijfer S, Foekens JA, et al: Association of an extracellular matrix gene cluster with breast cancer prognosis and endocrine therapy response. Clin Cancer Res 14: 5555-5564, 2008.

40. Cho BA, Cho NH, Seong SY, Choi MS and Kim IS: Intracellular invasion by Orientia tsutsugamushi is mediated by integrin signaling and actin cytoskeleton rearrangements. Infect Immun 78: 1915-1923, 2010.

41. Baker AM, Bird D, Lang G, Cox TR and Erler JT: Lysyl oxidase enzymatic function increases stiffness to drive colorectal cancer progression through FAK. Oncogene 32: 1863-1868, 2013.

42. Taubenberger AV, Bray LJ, Haller B, Shaposhnykov A, Binner M, Freudenberg U, Guck J and Werner C: 3D extracellular matrix interactions modulate tumour cell growth, invasion and angiogenesis in engineered tumour microenvironments. Acta Biomater 36: 73-85, 2016. 